rha Revue d'histoire du XIXe siècle

Société d'histoire de la révolution de 1848 et des

révolutions du XIXe siècle

$55 \mid 2017$

Les écoles du peuple à l'ère des révolutions

(1815-1880)

Claude PENNETIER, Jean-Louis ROBERT [dir.], Édouard Vaillant (1840-1915) de la Commune à l'Internationale

Paris, L'Harmattan, 2016, 210 p., $22 €$

Jean-Charles Buttier

CpenEdition

Journals

Édition électronique

URL : http://journals.openedition.org/rh19/5368

DOI : $10.4000 /$ rh 19.5368

ISSN : $1777-5329$

Éditeur

La Société de 1848

Édition imprimée

Date de publication : 1 décembre 2017

Pagination : 230-232

ISSN : 1265-1354

Référence électronique

Jean-Charles Buttier, «Claude PENNETIER, Jean-Louis ROBERT [dir.], Édouard Vaillant (1840-1915) de la Commune à I'Internationale», Revue d'histoire du XIXe siècle [En ligne], 55 | 2017, mis en ligne le 01 décembre 2017, consulté le 05 janvier 2021. URL : http://journals.openedition.org/rh19/5368 ; DOI https://doi.org/10.4000/rh19.5368

Ce document a été généré automatiquement le 5 janvier 2021.

Tous droits réservés 


\section{Claude PENNETIER, Jean-Louis ROBERT [dir.], Édouard Vaillant (1840-1915) de la Commune à l'Internationale}

Paris, L'Harmattan, 2016, 210 p., $22 €$

Jean-Charles Buttier

\section{RÉFÉRENCE}

Claude PENNETIER, Jean-Louis ROBERT [dir.], Édouard Vaillant (1840-1915) de la Commune

à l'Internationale, Paris, L'Harmattan, 2016, 210 p., $22 €$

1 Ce livre constitue les actes d'une journée d'études organisée en 2015 à l'occasion du centenaire de la mort de Vaillant (1840-1915) et permet de faire le récit de la longue carrière politique de celui qui fut membre de la Première Internationale avant d'être l'un des dirigeant.e.s de la Commune de Paris pour enfin œuvrer à l'unité socialiste en 1905. Les biographies antérieures de M. Dommanget (1956) et J. Howorth (1982) sont largement citées, le propos de l'ouvrage étant de les compléter par des éclairages thématiques. L'intérêt de cette écriture plurielle est d'interroger la transmission de la mémoire de personnages de premier plan comme Vaillant qui n'ont toutefois pas écrit d'autobiographie ou bien dont les archives encore conservées n'ont pas fait l'objet d'une valorisation. Ainsi, Elisabeth Badinter, l'une de ses descendant.e.s, insiste-t-elle dans sa préface sur un "défaut de transmission » familiale de la mémoire de son arrière-grand-père.

2 Claude Pennetier montre dans son chapitre intitulé " perspective biographique » que Vaillant a eu des engagements successifs. Il remarque notamment le fait que parmi le trio socialiste que formaient Jaurès, Guesde et Vaillant, la mémoire du troisième « céda le pas» (p. 18). L'une des hypothèses avancées est le «bellicisme effréné » (p. 23) de 
Vaillant en 1914 (l'historien cite alors Amédée Dunois) alors qu'il avait ardemment combattu la guerre jusque-là, ce changement de ton tardif jetant une ombre sur ses engagements précédents. Comme le font remarquer C. Pennetier et J.-L. Robert dans l'introduction générale, le parcours politique de Vaillant interroge la possibilité même de rendre compte d'une pensée foisonnante et complexe et rend incertaine la définition du vaillantisme.

3 La période de la Commune de Paris est fondatrice pour tenter de comprendre la personnalité et l'œuvre de Vaillant. Laure Godineau démontre la singularité des convictions politiques de celui qu'elle décrit comme "inclassable dès sa jeunesse » (p.32), dévoilant par la même occasion l'une des clés de compréhension de l'oubli relatif dans lequel est tombé Vaillant. L'historienne insiste sur le poids de la prise en charge par les acteurs et actrices historiques de leur propre postérité, notant ainsi qu'il n'a pas écrit de témoignage ou d'étude sur la Commune. Vaillant est pourtant un membre actif de la Commission exécutive. Jean-Louis Robert insiste justement sur le poids de cet engagement en consacrant un chapitre à l'œuvre de celui qu'il qualifie de «ministre » de l'enseignement et de la culture pendant la Commune. La courte période qui s'étend du 20 avril au 21 mai 1871 est cependant un «moment rare dans la vie de Vaillant qui n'eut que très rarement des responsabilités publiques » (p. 55). L'historien rappelle que le programme scolaire de la Commune que Vaillant tenta de mettre en œuvre était le fruit de discussion avec "L'éducation nouvelle, jeune société d'institutrices et d'instituteurs » à laquelle il appartenait. Ses membres étaient attaché.e.s à l'œuvre scolaire de la Révolution française prolongée par celle de la Seconde République et remise en cause par la loi Falloux de 1850. Les communard.e.s veulent inculquer aux élèves une morale civique républicaine qui rappelle les "élémens" (sic) de morale républicaine diffusés sous la Convention Nationale et le Directoire. Le socle de la politique scolaire de la Commune de Paris fut le décret séparant l'Église de l'État comme le rappelle J.-L. Robert. Les communard.e.s ont ensuite tenté de mettre en place une école fondée sur la science et la raison tout en défendant l'instauration d'une instruction intégrale conjuguant des contenus intellectuels et professionnels. Vaillant a aussi signé un décret instaurant l'égalité de salaire entre les instituteurs et institutrices. En charge de la culture, il se place dans la continuité des projets éducatifs de la Révolution française en voulant notamment faire des théâtres un «grand instrument de l'instruction publique » (p. 77).

4 Michel Pigenet, détaillant les premiers enracinements locaux du communard désormais amnistié, mène une étude de politique locale sur le département du Cher, terre d'élection de Vaillant. C'est ce que l'auteur appelle «l'étape berrichonne ». C'est une utile illustration de l'importance des réseaux locaux de sociabilité dans la construction d'une carrière politique. Jean-Numa Ducange étudie l'internationalisme de Vaillant qui présida la séance d'ouverture de la deuxième Internationale de 1889, conjointement avec Liebknecht. Marcel Turbiaux s'intéresse quant à lui à la carrière parisienne de Vaillant et surtout à son intérêt pour la question sociale alors que le socialiste est élu pour la première fois député en 1893 , et le reste jusqu'à sa mort. Revenant sur la création de la SFIO fondée en avril 1905, Gilles Candar rappelle le sort différent connu par les trois figures déjà évoquées qui ont été à l'origine de ce rapprochement. Pourtant, Vaillant a incarné un courant majeur du socialisme français et fut aussi surnommé le "grand-père» de la CGT fondée en 1895. Il appartient à Vincent Chambarlhac de conclure ce livre avec l'étude de la période de la Première guerre mondiale. C'est l'occasion d'analyser l'élaboration d'une historiographie, depuis les 
textes nécrologiques de 1915 jusqu'aux premières biographies très engagées avec par exemple celle de M. Dommanget en 1956. Cette véritable « suture entre les témoignages et les études historiques sur Vaillant» (p. 188) fut sollicitée selon E. Badinter par son propre père, Marcel Bleustein-Blanchet, qui n'appartenait pas lui-même à la famille du dirigeant socialiste. C'est un parcours politique marqué par la diversité et la complexité des engagements politiques que dessine cet ouvrage précieux qui permet de nourrir une réflexion sur les ressorts de l'oubli, même relatif. 